\title{
Association of Visual Objects and Olfactory Cues in Drosophila
}

\author{
Aike Guo ${ }^{1}$ and Karl Georg Götz ${ }^{2,3}$ \\ ${ }^{1}$ Laboratory of Visual Information Processing \\ Institute of Biophysics \\ Academia Sinica \\ Beijing 100101, China \\ ${ }^{2}$ Max Planck Institut für Biologische Kybernetik \\ D-72076 Tübingen, Germany
}

\section{Abstract}

Context-dependent preferences in a choice between an upper and a lower visual object of otherwise identical appearance were recorded during stationary flight of the fruitfly, Drosophila melanogaster, in a flight simulator. The test animal was held in a fixed orientation at the center of a wing-beat processor that converts attempted turns into counter-rotations of a surrounding cylindrical panorama. This allowed the fly to maneuver the preferred object into the actual direction of flight. Single flies were trained to avoid a course toward the visual object that had been associated with the aversive odor benzaldehyde (BAL).

Conditioned object avoidance was

investigated in different treatment groups by collective evaluation of the scores from 80 long-lasting flights ( $>1 \mathrm{hr}$ ). In addition to a significant cross-modal association, we found a striking long-term effect of transient exposure to BAL both in the embryonic and larval states. The preimaginal experience significantly increased the indifference to BAL in the adult flies. Disturbed vision does not account for this effect: Neither the perception nor the discrimination of the visual objects was significantly impaired in the investigated flies. Disturbed olfaction could explain the present results. Recently, however, preimaginal BAL uptake has been found to interfere directly with the retention of heat-shock-conditioned object avoidance.

${ }^{3}$ Corresponding author.

\section{Introduction}

The affinity of the fruitfly, Drosopbila melanogaster, for visual objects in its environment depends not only on the apparent contrast, size, and shape of an object, but also on the object-related sensory context in space and time. The fly uses specific strategies to optimize search behavior, for instance, (1) adaptation of the "spatial density" of random search to the spatial density of rewarding objects (Götz and Biesinger 1985b; Schuster and Götz 1994); (2) avoidance of immediate return to a just-visited object (Götz 1980, 1994; Bülthoff et al. 1982); (3) avoidance of remote objects, independent of their subjective size (Götz 1983, 1994; Schuster 1996); (4) avoidance of familiar objects without novel attributes (Dill and Heisenberg 1995); and (5) avoidance of objects that have been associated with punishing stimuli (Wolf and Heisenberg 1991; Dill et al. 1993, 1995; Guo et al. 1996). Each of these procedures requires an evaluation of the object-related sensory context. Strategies 3-5 have been studied under conditions of fixed flight in a flight simulator. Object avoidance can be of interest with respect to the genetic dissection of learning and memory in Drosopbila (for review, see Heisenberg 1989; Greenspan 1995) and to recent advances in the understanding of the prerequisites for long-term memory formation such as repeatedly interrupted training sessions, appropriate expression of cAMP response elementbinding (CREB) activator, and protein synthesis (Tully et al. 1994b; DeZazzo and Tully 1995; Greenspan 1995; Yin et al. 1995; Tully 1996).

In the present paper we describe the generation of associative context between an attractive visual object and the repellent odor of almonds, benzaldebyde (BAL), under operant conditions in a flight simulator. The fixation of an object in the

LEARNING \& MEMORY 4:192-204 @ 1997 by Cold Spring Harbor Laboratory Press ISSN1072-0502/97 \$5.00

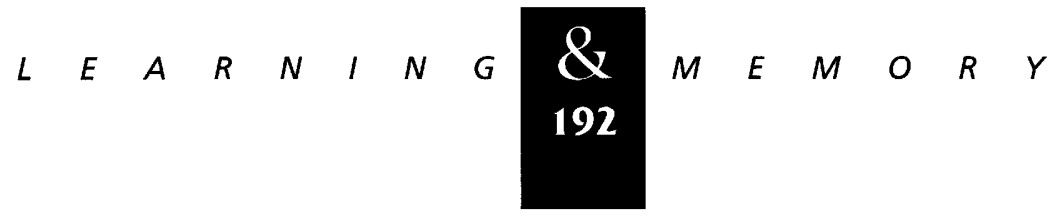


frontal visual field has been investigated at the neuronal level in larger flies (Reichardt and Guo 1986; for literature, see Egelhaaf et al. 1988). In Drosopbila, the processing of visual information (e.g., Götz 1968, 1987, 1994; Götz et al. 1979; Heisenberg and Wolf 1984; Wolf and Heisenberg 1991; Heide and Götz 1996) and the processing of olfactory information (for review, see Heisenberg 1989, 1994; Siddiqi 1991; Stocker 1994; de Belle 1995; Carlson 1996) have been investigated in considerable detail.

The olfactory system of Drosopbila consists of the antennal and maxillary sensilla, their afferents converging onto $\sim 35$ glomeruli within the antennal lobe on either side, and the major targets of the output of these lobes: the mushroom bodies and the lateral protocerebrum (Stocker 1994). The antennae contribute $\sim 90 \%$ and the maxillary palps of the proboscis $\sim 10 \%$ of the BAL-induced sensory input (Charro and Alcorta 1994). Odor-specific subsets of activated glomeruli have been mapped on autoradiographs of deoxyglucose uptake during unilateral olfactory stimulation. Attractants predominantly activated the ipsilateral subset; repellents such as BAL activated the ipsilateral and the contralateral subset of glomeruli (Rodrigues and Buchner 1984; Rodrigues 1988). This may explain why a stationarily walking Drosopbila continuously turns toward an attractant but rarely away from the repellent BAL (Borst and Heisenberg 1982). The discrimination of qualitative or quantitative differences between odor components has been analyzed in learning experiments (Borst 1983). By its course-control maneuvers in a flight simulator, Drosophila manages to stay in a virtual odor plume of fermenting banana. The fly uses its "operant activity" for a sophisticated retrieval of the desired sensation (Wolf and Heisenberg 1991). Ether anesthesia irreversibly disturbs searching for the source of this odor in the walking fly (Götz and Biesinger 1985a). The mushroom bodies (MBs) of Drosopbila are involved in olfactory discrimination and olfactory learning (Heisenberg 1994). Odor-specific deficiencies in the behavior of mutants with deranged $(m b d)$ or miniature $(m b m)$ MBs seem to support this conclusion (Heisenberg et al. 1985; de Belle and Heisenberg 1996). Moreover, chemical ablation of the MBs in the wild type impairs neither olfaction nor visual learning but abolishes the conditioned avoidance of BAL and other repellents (de Belle and Heisenberg 1994). In comparatively few successful experiments, thirdinstar larvae of Drosopbila learned to avoid shock- associated odors (Aceves-Piña and Quinn 1979; Heisenberg et al. 1985; Heisenberg 1989). After appropriate consolidation by repeatedly interrupted training sessions (spaced training), the memory even seemed to survive the reorganization of the nervous system during metamorphosis (Tully et al. 1994a). Unconditioned larvae are attracted by odorants such as BAL that become repellent for adult flies (Ayyub et al. 1990). A number of olfactory mutations have been identified that seem to interact with the processing of odorantspecific signals (for references, see Siddiqi 1991; Carlson 1996). Of those mutants, pentagon 7 ( $p \operatorname{tg} 7$ ) was found to be disturbed exclusively in its response to BAL (Helfand and Carlson 1989). Conversely, abnormal cbemical jump 6 (acj6) severely reduces the electrical response in the maxillary palps and most probably also in the antennae to all odorants tested except BAL, which seems to be processed separately (Ayer and Carlson 1992). Several other olfactory mutants show BAL-specific behavioral defects (Ayyub et al. 1990). Temporary transition to a poor diet has been found to gradually induce complete amnesia with a recovery time of four to five generations (Guo et al. 1996).

Here, we try to demonstrate the context-dependent avoidance of visual objects that had been associated with the repellent odor BAL. This requires the evaluation of the course-control maneuvers of Drosopbila during tethered flight in a simulator. The principle of the present paradigm has much in common with the approach to operant odor control described by Wolf and Heisenberg (1991). However, the technical realization, the experimental procedure, and the evaluation of the data deviate considerably from previous schemes and require a detailed account in the following section.

\section{Materials and Methods}

The present experiments were carried out on 2- to 3-day-old female fruitflies of the wild type Berlin from the stock held in Tübingen. The flies were raised, under standard conditions, on a medium comprising 80 grams of corn flour, 80 grams of malt extract, 22 grams of sugar beet syrup, 18 grams of dry yeast, 10 grams of soybean flour, 8 grams of agar, and 6 grams of propionic acid per liter of water. Careless nutrition can be detrimental to learning in Drosopbila (Guo et al. 1996).

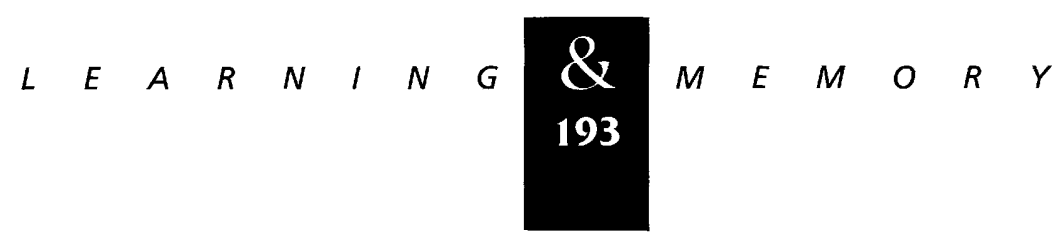




\section{OLFACTORY ADAPTATION}

Test flies were selected almost randomly from three simultaneously raised groups with a different history of previous exposure to BAL at a concentration of 0.05 grams per liter of medium: (1) The "no exposure" or $N$ group of naive flies had no previous exposure to BAL; (2) the "embryonic exposure" or E group of flies received BAL by maternal exposure only in their embryonic state. To obtain these flies, freshly hatched virgin females of the $\mathrm{N}$ group were transferred on day 1 to a tube containing $\sim 60 \mathrm{ml}$ of BAL-contaminated medium. Males of the $\mathrm{N}$ group were added to this tube on day 2 . The fertilized females were transferred on day 3 to the noncontaminated medium of an interim tube, where they were likely to lose most of the incorporated BAL by excretion, diffusion, and chemical transformation. From day 4 to day 5 , the flies were allowed to lay their eggs on the noncontaminated medium of another tube. The emerging E-group flies were collected from this tube. (3) The "larval exposure" or $L$ group of flies received BAL only in their larval state. These flies were obtained from the eggs laid by fertilized females of the $\mathrm{N}$ group during their temporary transfer to a tube containing BAL-contaminated medium. From hatching to pupation, the larvae fed on this medium. During this period of $\sim 10$ days, the contamination was repeatedly refreshed because of its gradual oxidation to benzoic acid. The emerging L-group flies were collected in a tube containing noncontaminated medium.

\section{FLIGHT SIMULATOR}

To investigate selection and tracking of a preferred visual object under stationary conditions, a flying Drosophila was held, by a $0.2-\mathrm{mm}$ wire glued to its head and thorax, in a fixed position and orientation at the center of a flight simulator. The fly was allowed to control, by its intended turns, the rotation of a surrounding panorama on a cylindrical screen about its vertical body axis (arrow in Fig. 1a). Intended turns can be derived directly from the moment of the flight force about this axis (Götz 1968; Götz et al. 1979; Heisenberg and Wolf 1984; Wolf and Heisenberg 1991; Dill et al. 1993, 1995) or, as in the present experiments, from a prominent variable of course control in Drosophila: the difference of the stroke amplitudes of the wings on either side. Details of the latter procedure have been described elsewhere (Götz et al.

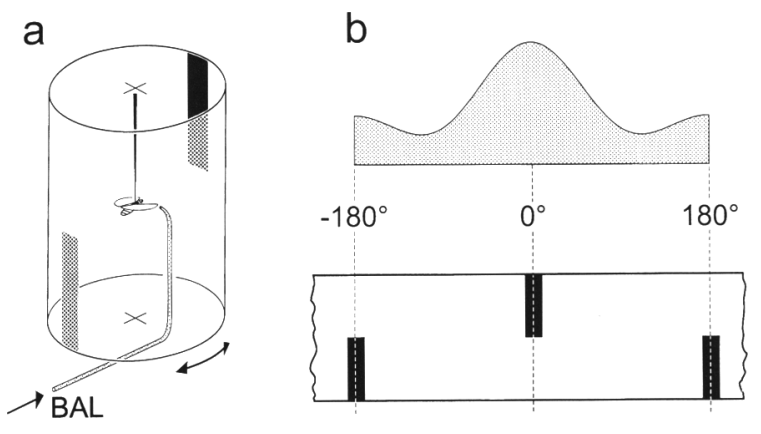

Figure 1: (a) Drosophila during fixed flight in a simulator. The intended turning, derived from the different widths of the wing stroke on either side, moves a panorama consisting of two identical but vertically displaced bar-shaped objects clockwise or counterclockwise around the fly. A course toward one of the objects is negatively reinforced by short pulses of the repellent odor BAL. (b) Schematic drawing of a histogram of the relative time spent on different directions of flight. The projection of the panorama shown below presents these directions in relationship to a spontaneously preferred reference object in the center. The example illustrates a fly-specific difference in the attraction of the two objects.

1979; Götz 1983, 1987, 1994; Heide and Götz 1996). To outline the essentials, it should be mentioned that the stroke amplitudes correspond to the maxima of the angular wing excursion at the end of the downstroke. The minima of this excursion are negligibly small: The wings touch or nearly touch each other at the end of the upstroke. Using infrared spotlights, the shadows of the beating wings were cast onto an optoelectric device. This device evaluates the excursions and returns signals proportional to the wing-beat amplitude on either side. The difference of these signals controls, by rotation of a circular transparency in a wide-angle projector on top of the simulator, the angular motion of its image on the cylindrical screen in Figure 1a. This allows the fly to maneuver the panorama into arbitrary angular positions to the direction of flight. The results obtained with the present method are comparatively insensitive to external forces such as the impact of a puff of odor on the tethered fly. Moreover, the actions of the two wings can be recorded separately and with the highest possible refresh rate of one measurement per stroke cycle $(-200 \mathrm{~Hz})$.

\section{CONDITIONING}

The panorama in the experiment depicted in

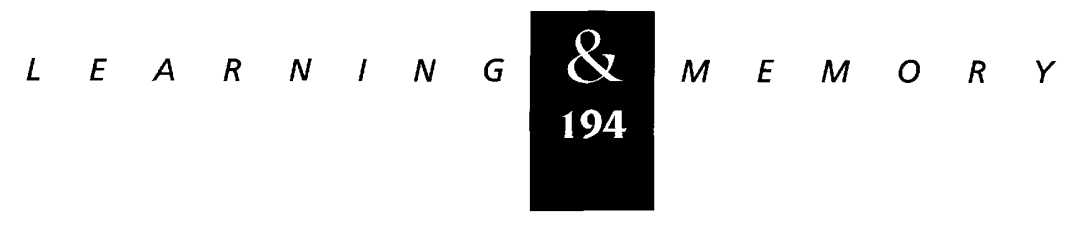


Figure $1 \mathrm{a}$ is comprised of two identical dark bars, each $\sim 60^{\circ}$ high and $30^{\circ}$ wide, presented in opposite positions on the upper and on the lower hemisphere of the visual field. During training, a course toward one of these bars can be punished by short pulses of repellent odor delivered through a nozzle of $0.8-\mathrm{mm}$ diameter positioned $\sim 3 \mathrm{~mm}$ anteroventrally from the head of the fly. To provide this odor, air was passed under a comparatively low pressure of $2-4 \mathrm{kPa}$ at a room temperature of $\sim 24^{\circ} \mathrm{C}$ through a gas washing bottle filled with a saturated aqueous solution of BAL ( $3 \mathrm{~g} / \mathrm{liter}$ ). The release of the odor was controlled by an electronic circuit acting on a miniature solenoid valve (LFAA 1207118H: LEE, Westbrook, CT). The valve opened periodically for $\sim 50-100 \mathrm{msec}$ at a rate depending on the actual angular deviation of the negatively reinforced ("punished") object from the fixed direction of flight. A maximum volley rate of $\sim 5 \mathrm{~Hz}$ was applied whenever the fly was heading toward this object. The rate decreased with the square of the angular deviation from this direction and was zero for deviations exceeding a range of up to $\pm 90^{\circ}$. The maximum delay time of a pulse of odor released by an attempted turn toward the object is inversely proportional to the volley rate: The range of the expected delay increases with the average angular deviation of the punished object from $\sim 20-200$ msec to $\sim 20-1000 \mathrm{msec}$. This is no serious limitation: The release of an odor pulse is always conditional upon the presence of the punished object in the frontal hemisphere of the fly. The response time of the valve and the time required for the propagation of a pulse of odor with an initial speed of $0.7 \mathrm{~m} / \mathrm{sec}$ from the nozzle to the fly (both $<5$ msec) were negligible in comparison to the expected delay. Pulses of odor-free air did not elicit perceivable reactions. This confirms earlier observations on stationarily walking flies (Borst and Heisenberg 1982).

\section{EVALUATION}

A tethered fruitfly controls the angular position of the panorama with respect to its fixed direction of flight by up to five distinct maneuvers per second (Heide and Götz 1996). These maneuvers induce angular displacements of the panorama at a speed of up to two revolutions per second. On average, the minimum time required for a transition between any two arbitrary positions of the panorama should be about $0.13 \mathrm{sec}$, the ratio of the average angular distance $\left(90^{\circ}\right)$ and the maximum angular speed $\left(-720^{\circ} / \mathrm{sec}\right)$. Actual angular positions were sampled every $0.50 \mathrm{sec}$, a time interval that exceeds the average time requirement for independent data by a factor of four. These considerations led us to assume statistical independence of successive samples in the present investigation. It could be argued that the flies try to stabilize actual angular positions of the panorama for quite a while, as described by the distribution of the "dwelling time" in a comparable experimental situation (Dill et al. 1995). However, dwelling is an active process. Stabilization of an angular position can be achieved only by continuous course control. Expectedly, the stabilization disappeared whenever the illumination of the panorama was switched off. The samples obtained under these conditions did not show dwelling or other conspicuous regularities. In spite of this evidence, an upper limit of the sampling error has been derived under the assumption that the length of the dwelling time rather than the sampling interval would account for the independence of the measurements. The quoted paper describes five experimental conditions that extend the average dwelling time. Evaluation of the corresponding data yields an upper time constant for dwelling of $6.0 \pm 1.2 \mathrm{sec}$ (S.D.) the equivalent of 12 sampling intervals. The improbable assumption about the influence of dwelling does not modify the means derived from the samples in the present experiments. However, the standard error of the means (S.E.M.) could increase under these conditions by a factor of up to 3.5 , the square root of 12 .

To evaluate the choice between different angular positions, the $360^{\circ}$ panorama was subdivided into 10 bins of $18^{\circ}$ on either side of an arbitrarily selected "reference object." In the standard experiment, a set of 50 samples was collected within $25 \mathrm{sec}$ of continuous flight in the simulator. For about the same time period, sampling was interrupted to convert the collected set of samples into a bistogram of the frequency of occurrence of the different angular positions (Fig. 1b) and to initiate the collection of the next set of samples. The intermission helps to randomize the initial angular position of the panorama. The results shown in Figures 3 and 4, below, refer to 13,790 stored histograms obtained by evaluating $96 \mathrm{hr}$ of flight from 80 flies. Continuation of flight during intermission of the sampling process adds to a total flight time of up to $\sim 200 \mathrm{hr}$.

A standard experiment consists of sequences

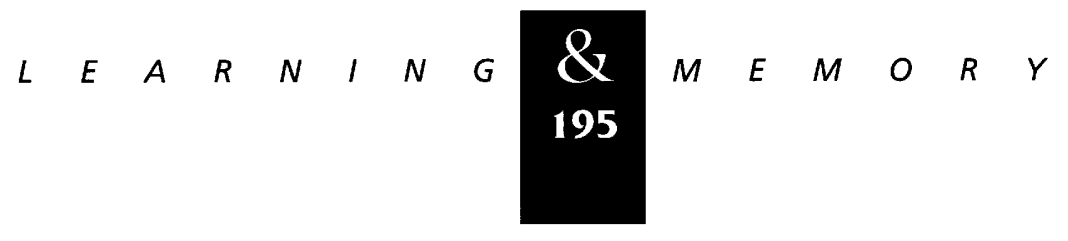


of four test periods (TE) and 12 training periods (TR). These sequences alternate in the order: pretest (TE0), first training (TR1), first test (TE1), second training (TR2), and second test (TE2). A standard period, or "slot," comprises the results of the histograms of five sets of samples that were collected within $2 \mathrm{~min}$ out of $\sim 4 \mathrm{~min}$ of continuous flight. To maintain this flight, the fly was fed to satiation with sugar water. This was done preferably just before a new training sequence. The average frequency of feeding was about one per hour. The allocation of the visual object associated with negative reinforcement by odor was exchanged at the beginning of the second training sequence (reversal conditioning).

In addition to the standard experiments described above, the results of two minor groups of experiments were included after appropriate redistribution of the available data according to the standard procedure: (1) experiments that were continued, after the last test TE2 and an ensuing second reversal of the object-odor allocation, in the repetitive order TR1, TE1, . . of the standard procedure and (2) pilot experiments that deviate from the time course of the standard experiment because of a larger number of samples/histogram and/or a lower or higher number of histograms/sequence: experiments with a histogram only for every other of the 12 slots of the training sequence TR2 in the $\mathrm{N}$ or L group account for a conspicuous "oscillation" of the corresponding results in Figures 4 and 5, below.

To avoid a biased selection from the available data, all flights of $>1 \mathrm{hr}$ duration were included in the collective evaluation of the long-term experiments on a treatment group of flies. It might be difficult to obtain equally long flights under the conditions of learning experiments with noxious heat shocks (Guo et al. 1996). As shown in Table 1, the minimum flight time is sufficient to complete the trials of the initial pretest (TE0) and the first training (TR1). Expectedly, however, quite a number of flies failed to fly long enough to complete the trials of the first test (TE1) and the following sequence of reversed-conditioning trials (TR2, TE2). The results obtained under these conditions relate to the behavior of an arbitrarily selected group of flies and do not allow us to assay the variability of acquisition and retention at the level of individual flies. This would require a reevaluation of the histograms of subgroups of flies with sufficient flight endurance.

\section{PERFORMANCE INDEX}

The actions of flies in a learning experiment are conveniently expressed by a performance in$\operatorname{dex}(P I)$ in the range $-1 \leqslant P I \leqslant+1$. The present experiments offer a choice between two different definitions:

1. PI of a sample is the cosine of the actual angle between reference object and direction of flight. PI $=+1$ indicates flight toward the reference object; PI $=-1$ indicates flight toward its counterpart. In most of the learning experiments described by $M$. Heisenberg and coworkers, the panorama consists of a regular $m$-fold repetition of the sequence of two different objects. For any positive integer $m$ (e.g., $m=2$ ), the present index PI is given by the cosine of $m$ times the actual angle between one of the reference objects and the direction of flight.

2. PI of a set of samples is the quotient $\left(n_{+}-n_{-}\right) /$ $\left.n_{+}+n_{-}\right)$derived from the number of samples for which the cosine of $m$ times the actual angle is either positive $\left(n_{+}\right)$or negative $\left(n_{-}\right)$. This quo-

Table 1: Number of flies completing up to five different trials during very long flights in the simulator

\begin{tabular}{lcccrc}
\hline & TE0 & TR1 & TE1 & TR2 & TE2 \\
\hline N group & 30 & 30 & 19 & 16 & 11 \\
E group & 25 & 25 & 25 & 23 & 23 \\
L group & 25 & 25 & 19 & 15 & $9^{*}$ \\
Approximate flight time (min) & 16 & 64 & 80 & 128 & 144 \\
Approximate sampling time (min) & 8 & 32 & 40 & 64 & 72 \\
\hline
\end{tabular}

*TE2 data only from flies with initial preference for the lower object.

$$
\begin{array}{lllllllllllllllll} 
& E & A & R & N & I & N & G & \begin{array}{l}
\boldsymbol{Z} \\
196
\end{array} & M & E & M & O & R & Y
\end{array}
$$


tient can be easily determined from the time a fly spends heading toward the respective sectors of the reference object $(+)$ or its counterpart (-). The resulting PI is commonly used to determine the performance of single flies (e.g., Wolf and Heisenberg 1991).

The almost equivalent definition of the type 1 PI in paragraph 1 has been selected because of minor advantages for the present attempt to establish the existence and the trend of a preference in a given set of flies. The cosine of the angular deviation from a reference object assesses preference by a continuously variable index. Each of the samples contributes immediately to the average PI. Accordingly, the standard error of the mean (s.E.M.) decreases with the square root of the number of statistically independent samples. It is not necessary to calculate PI according to paragraph 2 as a quotient for subsets of all-or-none events and to consider the size of these subsets in the calculation of the overall s.E.M..

Each of the means and s.E.M. values in Figure 4, below, comprises, on average, $\sim 6400$ samples of the type 1 PIs from up to 30 flies. The comparatively small error bars characterize the reliability, not the fluctuation, of the preference within an arbitrarily selected group of flies. For comparison, the samples were converted into all-or-none events $\left(\mathrm{n}_{+}, \mathrm{n}_{-}\right)$and processed, in subsets of five, according to the definition of the type 2 PI in paragraph (2). Expectedly, no conspicuous differences have been found between the means and s.E.M. values of corresponding PIs.

\section{SIGNIFICANCE TESTS}

The present study provides means and s.E.M. values of, within limits, continuously variable samples of PIs for three treatment groups (N, E, L). Each of these groups is subdivided according to the spontaneous preference for the upper or lower visual object in the pretest TEO. Within these groups or subgroups, a mean PI represents 1 out of either 12 slots of a training sequence (TR1, TR2) or 4 slots of a test sequence (TE0, TE1, TE2). To assay significances, each of the means is treated as one individual "observation." By neglecting the statistics of the great number of underlying samples, the results can be used to obtain conservative estimates of the reliability of conditioned object avoidance within an arbitrarily selected population of flies. Normal distribution of the samples $(P \leqslant 0.05)$ either in 27 of the 30 sequences from the subgroups for different object preferences in the pretest or in all of the 15 sequences from the pooled subgroups seemed to allow us the omission of an arcsine transformation of the raw data in the present tests. All pairwise comparisons are based on a priori hypotheses and observations and are therefore "planned." To account for nonorthogonal comparisons, the statistical tests applied (ANOVA, Student-NewmanKeuls) estimate the experimentwise type 1 error rate.

\section{Results}

Figure 1a illustrates the investigation of a choice between two visual objects in a flight simulator. The intended turns of the fixed fly control the rotation of the panorama about its vertical axis. Martin Heisenberg and his coworkers have shown how the fly learns to avoid a course toward objects that have been associated with noxious heat shocks. In the present experiments, Drosopbila was trained to avoid a course toward one of the bar-shaped objects that was associated with pulses of the repellent odor BAL. Figure $1 \mathrm{~b}$ shows a projection of the panorama. A "typical" histogram on top illustrates the time spent by a fly on the different directions of flight. The maxima indicate the preferred orientation. The peaks at $0^{\circ}$ and $\pm 180^{\circ}$ indicate the attraction of the objects in comparison to the void areas of the panorama owing to object perception. The difference between the peak at $0^{\circ}$ and the peak at $\pm 180^{\circ}$ indicates the individual preference of one object over the other, thus representing object discrimination. Here, we describe the results of odor-conditioned object avoidance in three differently treated groups of flies.

\section{AVOIDANCE OF THE AVERSIVE ODOR BAL}

Figure 2 shows, in temporal sequence from front to back, the results obtained by evaluating 37 hr of flight in 30 flies from the untreated $\mathbf{N}$ group. This amounts to $\sim 2 \mathrm{~min}$ of sampling time in $\sim 4 \mathrm{~min}$ of continuous flight per histogram and fly. During the initial trials of the pretest (TE0), the naive flies spontaneously prefer either the upper object (19 flies; histograms on the left) or the lower object (11 flies; histograms on the right). Attempted flight

$$
\text { ………………… }
$$


Figure 2: Odor-induced sequence of avoidance and acceptance of the initially preferred upper object (19 flies, histograms at left) or lower object (11 flies, histograms at right). (TEO) Pretest: the reference object in the center coincides with the peaks of initial preference. (TR1, TE1) First training and test: sustained avoidance of the reference object if its selection is associated with repellent odor. (TR2, TE2) Second training and test: sustained acceptance of the reference object if selection of the opposite

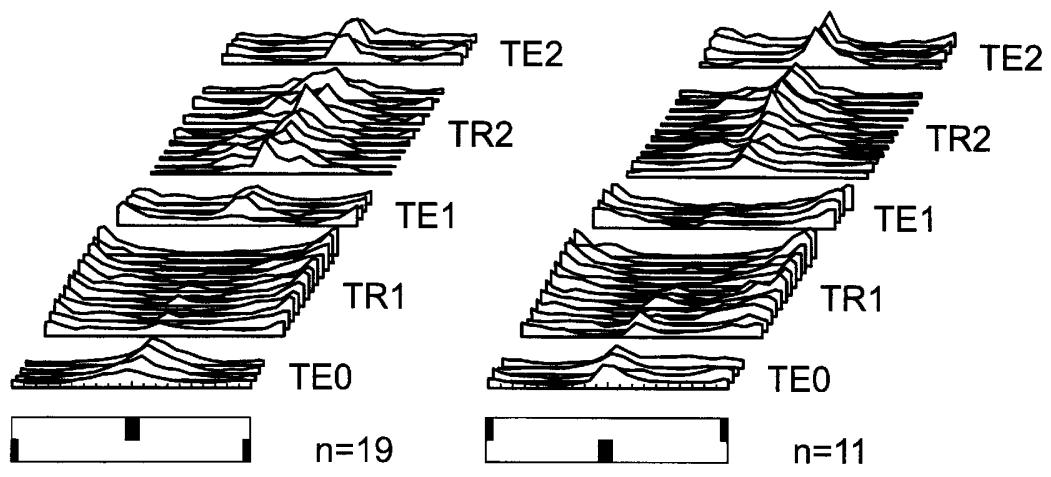
object is associated with repellent odor (reversal conditioning). The time interval of fixed flight is $\sim 4$ min per histogram and fly, or $\sim 2.4 \mathrm{hr}$ for the complete sequence of 36 trials. Statistical comparison of the results shows that the retention of the acquired preference is independent of the initially preferred object.

toward the preferred object is punished by short pulses of the repellent odor during the trials of the first training (TR1). The histograms show the avoidance of this object. The aftereffect of this treatment is determined during the trials of the first test (TE1). The histograms show the retention of the acquired avoidance. Then, we reversed the allocation of the stimuli: Attempted flight toward the "other" object is punished and avoided during the trials of the second training (TR2). The aftereffect, the retained avoidance, is determined during the trials of the second test (TE2). Comparison of the responses of single flies shows individual differences. However, conditioned object avoidance could be seen in almost all of the experiments. Moreover, the effect of retained avoidance is invariant to the exchange of the upper and the lower object. Using a representation where each of the histograms is described by a PI, where $-1 \leqslant \mathrm{PI} \leqslant+1$ that grows with the central peak (see the corresponding section in Materials and Methods), a Student-Newman-Keuls test (SNK test, $P \leqslant 0.05$ ) confirmed the expected relationship for retained avoidance $\left(\mathrm{PI}_{\mathrm{TEI}}<\mathrm{PI}_{\mathrm{TEO}}\right)$ and retained avoidance after reversal $\left(\mathrm{PI}_{\mathrm{TE2}}>\mathrm{PI}_{\mathrm{TE1}}\right.$ ) for each of the subgroups in Figure 2. We pooled these subgroups because of the similarity of these results.

\section{ADAPTATION TO BAL}

Further experiments in the flight simulator suggest that early exposure to BAL in the medium increases the "indifference" toward this odor in the adult flies. Figure 3 shows, from left to right, the responses of three different groups of flies that were able to fly for $>1 \mathrm{hr}$ in the simulator. (1) The
$\mathrm{N}$ group of 30 naive flies (total sampling time of 37 hr) had not been exposed to BAL before the experiment. This group is represented by the pooled results in Figure 2. (2) The E group of 25 flies (total sampling time of $31 \mathrm{hr}$ ) was exposed to BAL in their embryonic state as eggs in the ovaries of their mothers. (3) The L group of 25 flies (total sampling time of $28 \mathrm{hr}$ ) was exposed to BAL in their larval state.

The number of flies with initial preference for the upper (lower) object was 19 (11) in the $\mathrm{N}$ group, 12 (13) in the E group, and 10 (15) in the $\mathrm{L}$ group. Corresponding results have been pooled in analogy to the treatment of the data from the naive flies.

Preimaginal exposure to $\mathrm{BAL}$ in the medium decreases both the avoidance of the punished object during training and the retention of that avoidance during the test. This is more easily seen in a representation of the means and S.E.M. values of the PI in Figure 4. The bar graphs show, on the left, the spontaneous preference for either the upper or the lower visual object during the pretest (TE0) in each of the three groups. In these and the following trials, PI $>0$ is always assigned to the selection of the initially preferred object. During the first training (TR1), the previously preferred object is strongly avoided in the $\mathrm{N}$ group. The avoidance is reduced in the $\mathrm{E}$ group and is missing in the $\mathrm{L}$ group. Retained avoidance in the corresponding first test (TE1) is significant only in the $\mathrm{N}$ group and is missing to a significant degree in the other groups. This also seems to be valid for the second training (TR2) and second test (TE2) under reversed training conditions. SNK tests $(P \leqslant 0.05)$ for the three groups proved the significance of the following relations: $\mathrm{N}$ group: $\mathrm{PI}_{\mathrm{TR} 1}<\mathrm{PI}_{\mathrm{TE} 1}<\mathrm{PI}_{\mathrm{TE} 0}$,

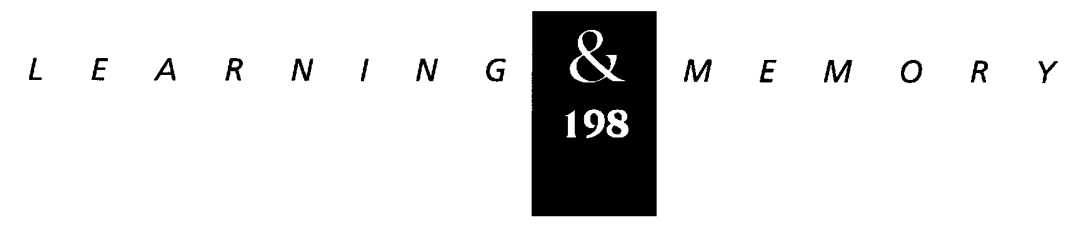




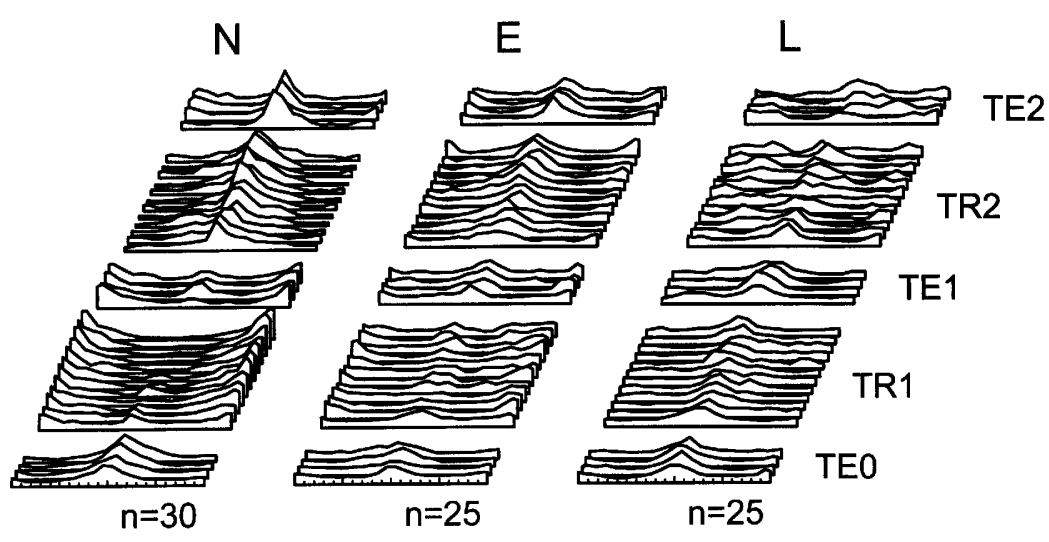

Figure 3: Effect of preimaginal exposure to BAL on the pooled results of the two sets of experiments described in the legend to Fig. 2. (N) Results of Fig. 2 for comparison: contribution of 30 naive flies that had not been exposed to BAL before the experiment. (E) Contribution of 25 flies exposed to BAL during their embryonic development. (L) Contribution of 25 flies exposed to BAL during their larval development. Note, from left to right, the gradual decrease in the avoidance of the punished object with increasing intensity of the preimaginal exposure to BAL in the medium.
$\mathrm{PI}_{\mathrm{TR} 2}>\mathrm{PI}_{\mathrm{TE} 2}>\mathrm{PI}_{\mathrm{TR} 1}$, and $\mathrm{PI}_{\mathrm{TR} 1}=-\mathrm{PI}_{\mathrm{TR} 2}$, that is, avoidance to about the same degree for TR1 and TR2 and retained avoidance for TE1 and TE2. E group: $\mathrm{PI}_{\mathrm{TR} 1}<\mathrm{PI}_{\mathrm{TE} 0}, \mathrm{PI}_{\mathrm{TR} 2}>\mathrm{PI}_{\mathrm{TE} 1}$, and $\mathrm{PI}_{\mathrm{TEO}}=\mathrm{PI}_{\mathrm{TE} 1}=\mathrm{PI}_{\mathrm{TE} 2}$, that is, avoidance for TR1 and TR2 but no retention of avoidance for TE1 and TE2. L group: $\mathrm{PI}_{\mathrm{TR} 1}=\mathrm{PI}_{\mathrm{TE} 1}=\mathrm{PI}_{\mathrm{TEO}}$, that is, no avoidance for TR1 and no retention of avoidance for TE1. Increasingly irregular flight as a consequence of the extremely high dose of aversive odor received in this group is likely to explain the apparently negative avoidance for TR2 and negative retention of avoidance for TE2 according to $\mathrm{PI}_{\mathrm{TE} 2}<\mathrm{PI}_{\mathrm{TR} 2}<\mathrm{PI}_{\mathrm{TE} 1}$.

A three-way ANOVA testing the effects of treatment, type of trials, initial preference, and their interactions confirmed the influence of preimaginal BAL exposure on the BAL responses of the adult flies $[F(2,183)=30.5, P<0.0001]$.

The punishment received by a fly during the training is measured in odor pulses per minute. Figure 5 shows a gradual reduction of this rate to a comparatively low residuum of "curiosity" in the N group. The other groups seem to "tolerate" much higher levels of punishment. The L group appears to be almost indifferent to this treatment. This is not necessarily a physiological explanation. The excessive level of punishment received during a standard experiment may account for the increasingly irregular flying behavior observed after $\sim 80$ min of flight in the $\mathrm{L}$ group. However, all of the flies survived the heavy dosage of aversive odor, in contrast to the experience with heat shock as a negative reinforcer. The gradual increase in the pulse rate with the intensity of preimaginal exposure to BAL has been verified (SKN test, $P \leqslant 0.001$ ) for the training before the reversal (TR1) according to $\mathrm{N}<\mathrm{E}<\mathrm{L}$ and for the training after the reversal according to $\mathrm{N}<\mathrm{E}=\mathrm{L}$.

Several explanations may account for the effects of early exposure to BAL: The repellent could interfere with perception, avoidance, and conditioning of avoidance in a most unspecific way. However, the relevant functions of the visual system are obviously not significantly disturbed. This is shown in Figure 6. The symbols indicate the classification of the flies within the $\mathrm{N}, \mathrm{E}$, and $\mathrm{L}$ group according to their spontaneously preferred object. The means and s.D. values of the "harmonics" explained in the figure legend represent the perception of the two objects (upper diagram) or the discrimination between these objects (lower diagram). The increase in the intensity of early exposure to BAL from the $\mathrm{N}$ group on the left to the $\mathrm{L}$ group on the right is not accompanied by a corresponding gradual decrease in these components.

\section{Discussion}

\section{OBSERVATIONS}

The present attempt to develop a long-term assay for cross-modal association of visual and olfactory cues in Drosopbila was motivated by the interest in the confluence of different sensory modalities in the insect nervous system. Honeybees combine visual input from the optic lobes and olfactory input from the antennal lobes in their MBs in a complex scheme of context-dependent modal integration (Gronenberg 1986; Hammer and Menzel 1995). Some of the neurogenetic means and ways mentioned in the Introduction could help to address such problems in Drosopbila.

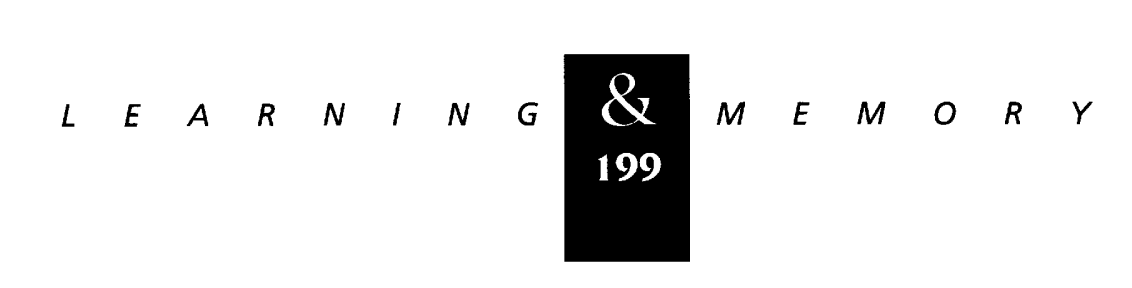




$$
\text { Performance Index PI ( } \pm \text { SEM) }
$$
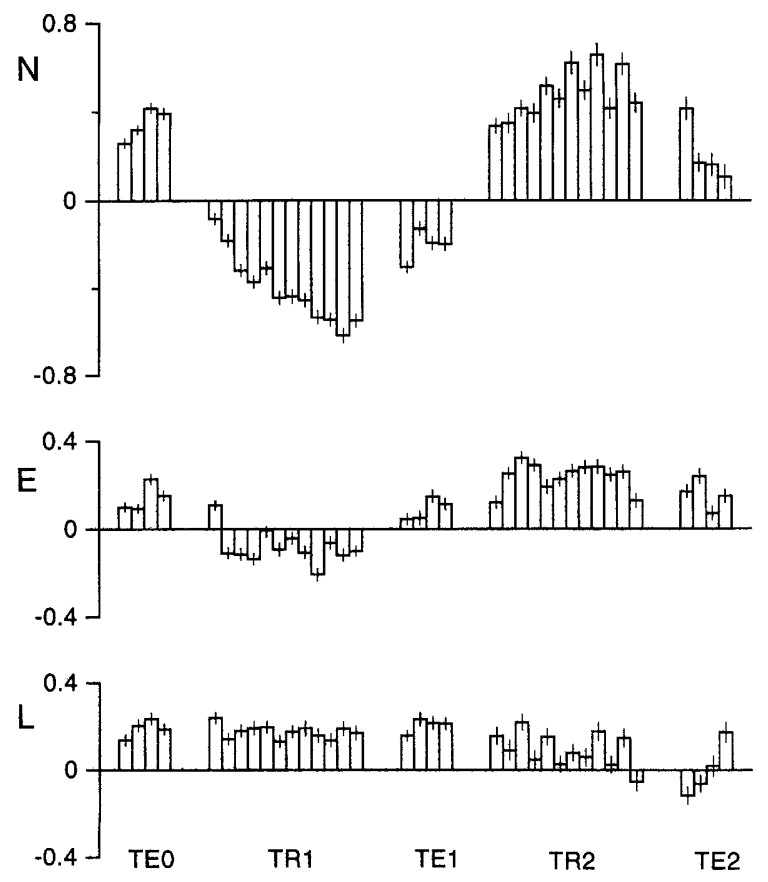

Figure 4: Time course of the $\mathrm{Pl}$ in the range of $-1 \leqslant P I \leqslant+1$, describing the acceptance of the initially preferred reference object for the $N, E$, and $L$ groups of flies in Fig. 3. The means and the comparatively small S.E.M. values for the different conditions of training (TR1, TR2) and test (TE0, TE1, TE2) were derived from a total of 690,000 essentially independent samples in 13,790 recorded histograms. Avoidance of the punished object, retention of the avoidance, and reversibility of the retention are significant effects of the $\mathrm{N}$ group. In comparison, the avoidance is significantly reduced in the $\mathrm{E}$ group and missing in the $L$ group, whereas the retention and the retention after reversal of the conditioned object avoidance are missing in the $\mathrm{E}$ and $\mathrm{L}$ groups. To diminish the risk of a systematic error owing to a slow drift in the performance of our strain, the test flies were selected in a random-like sequence from simultaneously raised flies of the three groups. The high dosage of odor received by the $L$ group seems to account for irregular flight in the second half time.

The odor-conditioned object discrimination found in the present experiments suggests a significant association between the aversive odor of BAL and particular features of the two visual objects. The responses of the naive flies ( $N$ group) shown in the panel on top of Figure 4 led us to the following conclusions: (1) A single distinctive parameter, the elevation of the otherwise identical objects in the visual field of the fly, is sufficient for the formation of cross-modal links; (2) the group of naive flies avoids the aversive odor during the training (TR1, TR2) and continues to avoid the visual object associated with this odor during the following test (TE1, TE2); and (3) continuation of the training for an exceedingly long period of $\sim 48 \mathrm{~min}$ does not seem to diminish the efficiency of the aversive odor.

The last result contrasts with the novel effect of preimaginal BAL treatment shown in the lower panels of Figure 4. (1) Early exposure to BAL in the larval state (L group) and most likely even in the embryonic state (E group) significantly increases the indifference toward this odor in the adult flies. (2) In comparison with heat shock experiments (e.g., Guo et al. 1996), Drosopbila seems to tolerate long periods and high pulse rates of BAL punishment. Only some flights of the $\mathrm{L}$ group became increasingly irregular after $\sim 1 \mathrm{hr}$. This may be explained by the threefold increase in the average rate of odor pulses received by the BAL-indifferent L flies, if flight toward the initially preferred object is punished (TR1 in Fig. 5).

\section{Odor pulses / $\min ( \pm$ SEM)}


Figure 5: Time course of the average number of odor pulses per minute blown during the training toward an average fly of the N, E, or L group in Figs. 3 and 4 . The means \pm S.E.M. indicate the intensity of attempted flight toward the punished object. For obvious reasons, it is more difficult to avoid the initially preferred object (TR1) than to avoid the initially rejected object (TR2). The flies of the $\mathrm{N}$ group seem to maintain a residuum of "curiosity" for the punished object over extended periods of successful training. The apparent indifference towards the repellent odor increases significantly with the preimaginal uptake of BAL.

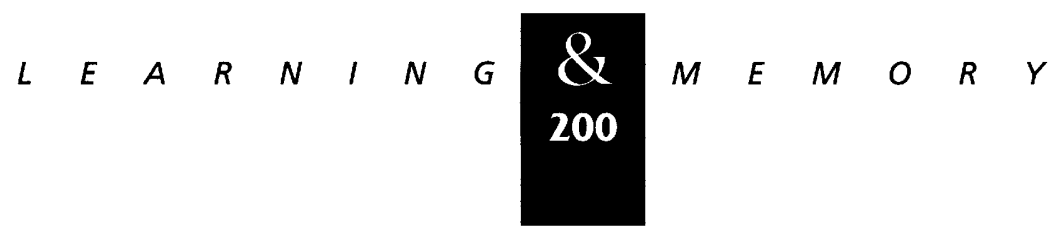




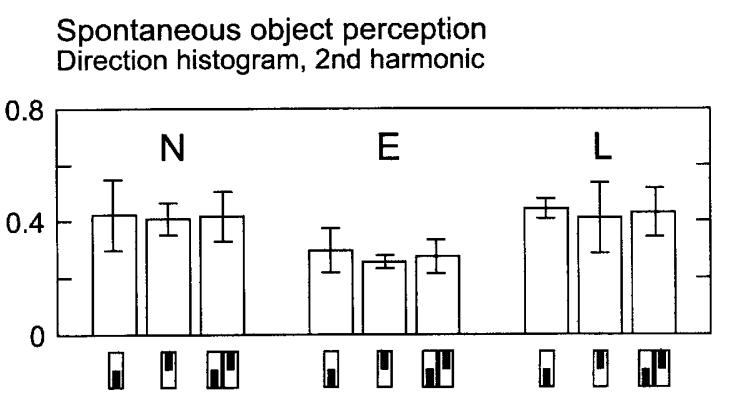

Spontaneous object discrimination Direction histogram, 1st harmonic

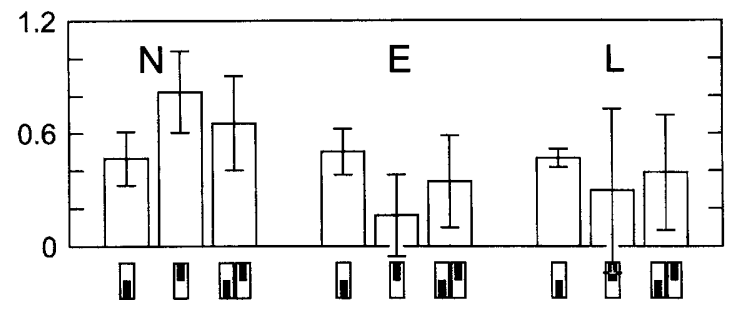

Figure 6: Two essential constituents of the pretest (TEO) histograms from the flies of the $N, E$, and $L$ groups in Fig. 3 were derived by Fourier analysis. The amplitude of the second harmonic with its peaks at the positions of the spontaneously preferred and the spontaneously rejected objects $\left(0^{\circ}\right.$ and $\left.\pm 180^{\circ}\right)$ represents the perception of the two objects. The amplitude of the first harmonic with its peak at the position of the spontaneously preferred object $\left(0^{\circ}\right)$ and its trough at the position of the spontaneously rejected object $\left( \pm 180^{\circ}\right)$ represents the discrimination of the two objects. The corresponding diagrams show the means \pm S.D. of these amplitudes in units of the normalized mean value of the histograms. The results are given in blocks of three bars. Two bars show the contributions of flies with a preference for either the lower or the upper object. The third bar combines these contributions. Neither the perception nor the discrimination of the two objects is significantly impaired by the early uptake of response-inhibiting amounts of the repellent BAL.

\section{RESTRICTIONS AND RESERVATIONS}

The data of the present long-term experiments on cross-modal association were derived by superposition of all available scores from unusually long individual flights of $>1 \mathrm{hr}$ in the simulator. However, not all of the flights were long enough to pass the five different sequences of trials in a complete standard program. The means and s.E.M. values in Figures 4 and 5 show the results of this procedure. The conservative statistical treatment of the means as single observations proves the main conclusions for a treatment group of flies according to Table 1 .
The attempt to assay the variability of acquisition, retention, extinction, and other important parameters of learning and memory for individual flies would have required the exclusion of the considerable amount of data obtained from long-lasting but incomplete flights. The time scale of the present long-term experiments should be shortened to obtain the appropriate data for a single-fly analysis of the new effects.

\section{RETENTION OF CONDITIONED PREFERENCES IN A NEW PARADIGM}

A number of inquiries are desirable for further investigations.

1. Numerous learning experiments with Drosopbila in a flight simulator use heat shocks instead of repellent odor to reinforce object avoidance (Wolf and Heienberg 1991; Dill et al. 1993, 1995; Dill and Heisenberg 1995; Guo et al. 1996). By analogy to these experiments, one might expect that operant conditioning applies to the present experiments as well. However, this remains to be verified by a failure of the flies of a "yoked" group to learn by passively witnessing the playback of pattern motion and odor reinforcement recorded in a successful training experiment (Wolf and Heisenberg 1991).

2. The half time of extinction remains to be determined. In one of their learning experiments, Dill et al. (1993) found that a delay of $20 \mathrm{hr}$ was not long enough to extinguish the conditioned response. On the other hand, the memory appeared to be comparatively volatile during an ongoing test. The present observations of the behavior in the flight simulator suggest a balance between "reluctance" and "curiosity" about the previously punished object. The reluctance is given up as soon as a hesitantly attempted approach of this object turns out to be safe (active extinction of memory). This may explain why, in Drosopbila, it is comparatively difficult to assess retention in extended tests and comparatively easy to alternate successfully between the objects conditioned for avoidance as shown in Figure 2. Flies of the $\mathrm{N}$ group seem to maintain their residuum of curiosity about the punished object over extended periods of successful training (Fig. 5). This might be advantageous for foraging solitary insects that do not

$$
\text { n....... }
$$


benefit by communication and cooperation from the exploratory efforts of an entire colony. The recently discovered scheme of controlled long-term memory formation in Drosopbila (Tully et al. 1994b; DeZazzo and Tully 1995; Yin et al. 1995) suggests an adaptive selection of input to be stored according to species-specific requirements.

3. The otherwise identical bar-shaped objects of the panorama in Figure 1a can be distinguished only by their appearance either in the upper or in the lower hemisphere of the visual field. The projection of a seamless panorama onto the inner side of a stationary screen surrounding the fly most likely excludes object distinction according to other cues. A verification of this statement would require evidence that the flies fail to show conditioned object discrimination in an experiment with identical objects of equal elevation (Wolf and Heisenberg 1991).

\section{DEVELOPMENT AND RETENTION OF ODOR INDIFFERENCE}

With due caution, it can be concluded from the results in Figures 4 and 5 that early exposure to BAL in the larval state, and even in the embryonic state, irreversibly increases the indifference of the adult fly toward this odor. Retention of avoidance is missing and is not likely to be expected under these conditions. However, the BAL indifference of the $\mathrm{E}$ and $\mathrm{L}$ groups in Figure 4 is possibly not the only reason for the lack of retention. In a recent series of still unpublished experiments, L. Liu, S.Z. Xia, L. Jia, C.H. Feng, and A.K. Guo repeated the described exposure of wild-type Berlin flies to BAL at different stages of their development. In the subsequent retention tests, these investigators measured the ability of the flies to avoid visual objects that were associated, during the training, with heat shocks (Wolf and Heisenberg 1991; Dill et al. 1993, 1995; Dill and Heisenberg 1995; Guo et al. 1996) instead of the repellent odor used in the present experiments. A BAL-induced increase in the indifference toward heat shocks during the acquisition trials was not found under these conditions. Nevertheless, the exposure to BAL in the embryonic, the larval, and the adult state significantly diminished or suppressed the avoidance of the previously punished object. It took about three generations of growth on a noncontaminated medium to fully reestablish the ability to learn. This effect can- not be explained by disturbed olfaction: At least in the heat shock experiments, BAL seems to interfere directly with the retention of the previously acquired experience. Apparent amnesia as a consequence of disturbed vision can be dismissed as well: The results in Figure 6 exclude substantial inhibiting effects of the repellent odor on both perception and discrimination of the objects used in the present paradigm.

The complete suppression of the avoidance of a punished object in the training and test sequences of the $L$ group requires a reliable transfer of chemical experience through the metamorphosis of Drosopbila. Is there any other evidence of the feasibility of conveyance between different stages of development? In a few successful experiments, third-instar larvae learned to avoid shockassociated odors (Aceves-Piña and Quinn 1979; Heisenberg et al. 1985; Heisenberg 1989). Using repeatedly interrupted training sequences (spaced training) for long-term memory consolidation in the larvae, the conditioned response was observed, after 8 days, in at least one set of adult flies (Tully et al. 1994a).

The recovery of early experience in the adult state is more difficult to accept for the reduction of BAL avoidance in the E group. A transfer of chemical experience from an egg to a succession of three larval instars followed by metamorphosis into the imago seems difficult to believe and raises questions as to the validity of our evidence. First of all, the reliable statistical significance of the differences in the performance of N, E, and L flies does not necessarily prove the influence of early exposure to BAL. The differences could also result from unknown parameters that are not sufficiently balanced in the three groups. Most of the laboratories working on learning and memory in Drosopbila share the observation of an occasional decline in the performance index. In these cases, impaired learning is tentatively attributed to changes in nutrition or handling (Guo et al. 1996), to infestation with parasites, or to seasonal parameters. To reduce the risk of such effects, we selected the test flies in a random-like sequence from the simultaneously raised $\mathrm{N}, \mathrm{E}$, and $\mathrm{L}$ group of otherwise identically treated flies of the same wild-type stock. Nevertheless, the regular oscillation of the results obtained in the $\mathrm{N}$ and $\mathrm{L}$ group during the second training sequence (TR2 in Figs. 4 and 5) is attributed to an unidentified shift in the performance of two sets of equally treated flies that contributed histograms either for every slot or for every other

$$
\text { ….... 圆 }
$$


slot of the training sequence (see the section on evaluation in Materials and Methods). However, the possibility of an influence of the conditions encountered in the egg stage on the behavior of the adult flies has been repeatedly demonstrated in a series of experiments by Kim et al. (1996). The authors found that mixed culturing of eggs from different semispecies of the Drosopbila paulistorum group weakened selectively the mutual attraction of adult females and males from different semispecies. A transfer of chemical experience from the earliest to the latest stage of development could explain these results.

So far, a valid explanation for the long-term effect of BAL indifference induced by early exposure to this odorant is missing and requires further experiments. It appears worthwhile to explore the unknown physiological or neuronal basis behind this effect. Chemical ablation of the MBs (de Belle and Heisenberg 1994) and the inclusion of BALspecific mutations (Carlson 1996) offer promising possibilities for future investigations.

\section{Acknowledgments}

We are grateful to Michael Renner for patiently processing and condensing the unusual amount of experimental data, to Steven de Belle for his valuable advice and his efficient help with the execution of the statistical tests on a PC, to Martin Heisenberg, Tim Tully, and our two anonymous referees for their most appreciated comments and suggestions, and to Diane Blaurock for her experienced support in the preparation of the manuscript. This project was funded in part by a Foreign Guest Scientist stipend of the Max Planck Society and the National Science Foundation of China granted to A.K.G.

The publication costs of this article were defrayed in part by payment of page charges. This article must therefore be hereby marked "advertisement" in accordance with 18 USC section 1734 solely to indicate this fact.

\section{References}

Aceves-Piña, E.O. and W.G. Quinn. 1979. Learning in normal and mutant Drosophila larvae. Science 206: 93-96.

Ayer, R.K.J. and J. Carlson. 1992. Olfactory physiology in the Drosophila antenna and maxillary palp: acj6 distinguishes two classes of odorant pathways. J. Neurobiol. 23: 965-982.

Ayyub, C., J. Paranjape, V. Rodrigues, and O. Siddiqi. 1990. Genetics of olfactory behaviour in Drosophila melanogaster. J. Neurogenet. 6: 243-262.

Borst, A. 1983. Computation of olfactory signals in Drosophila melanogaster. J. Comp. Physiol. 152: 373-383.

Borst, A. and M. Heisenberg. 1982. Osmotropotaxis in Drosophila melanogaster. J. Comp. Physiol. 147: 479-484.
Bülthoff, H., K.G. Götz, and M. Herre. 1982. Recurrent inversion of visual orientation in the walking fly, Drosophila melanogaster. J. Comp. Physiol. 148: 471-481.

Carlson, J.R. 1996. Olfaction in Drosophila-from odor to behavior. Trends Genet. 12: 175-180.

Charro, M.J. and E. Alcorta. 1994. Quantifying relative importance of maxillary palp information on the olfactory behavior of Drosophila melanogaster. J. Comp. Physiol. A 175: 761-766.

de Belle, S. 1995. Drosophila mushroom body subdomains: Innate or learned representations of odor preference and sexual orientation? Neuron 15: 245-247.

de Belle, S. and M. Heisenberg. 1994. Associative odor learning in Drosophila abolished by chemical ablation of mushroom bodies. Science 263: 692-695.

1996. Expression of Drosophila mushroom body mutations in alternative genetic backgrounds: A case study of the mushroom body miniature gene $(\mathrm{mbm})$. Proc. Natl. Acad. Sci. 93: 9875-9880.

DeZazzo, J. and T. Tully. 1995. Dissection of memory formation-from behavioral pharmacology to molecular genetics. Trends Neurosci. 18: 212-218.

Dill, M. and M. Heisenberg. 1995. Visual pattern memory without shape recognition. Phil. Trans. R. Soc. Lond. B 349: 143-152.

Dill, M., R. Wolf, and M. Heisenberg. 1993. Visual pattern recognition in Drosophila involves retinotopic matching. Nature 365: 751-753.

1995. Behavioral analysis of Drosophila landmark learning in the flight simulator. Learn. \& Mem. 2: 152-160.

Egelhaaf, M., K. Hausen, W. Reichardt, and C. Wehrhahn. 1988. Visual course control in flies relies on neuronal computation of object and background motion. Trends Neurosci. 11: 351-358.

Götz, K.G. 1968. Flight control in Drosophila by visual perception of motion. Kybernetik 4: 199-208.

1980. Visual guidance in Drosophila. In Development and neurobiology of Drosophila (ed. O. Siddiqi, P. Babu, L.M. Hall, and J.C. Hall), pp. 391-407. Plenum, New York, NY.

1983. Genetischer Abbau der visuellen Orientierung bei Drosophila. Verh. Dtsch. Zool. Ges. 1983: 83-99.

1987. Course-control, metabolism and wing interference during ultralong tethered flight in Drosophila melanogaster. J. Exp. Biol. 128: 35-46.

1994. Exploratory strategies in Drosophila. In Neural basis of behavioural adaptations (ed. K. Schildberger and N.

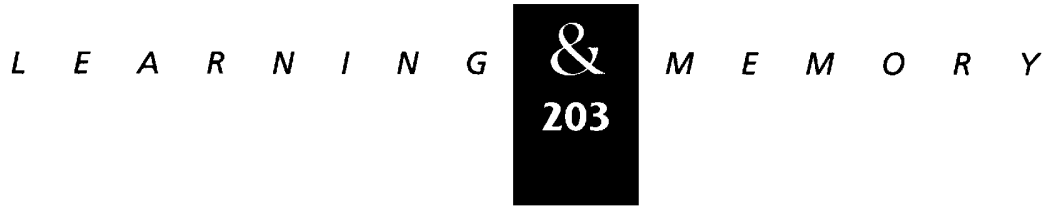


Elsner), Fortschritte der Zoologie 39, pp. 47-59. G. Fischer, Stuttgart, Germany.

Götz, K.G. and R. Biesinger. 1985a. Centrophobism in Drosophila melanogaster. I. Behavioral modification induced by ether. J. Comp. Physiol. A 156: 319-327.

1985b. Centrophobism in Drosophila melanogaster II. Physiological approach to search and search control. J. Comp. Physiol. A 156: 329-337.

Götz, K.G., B. Hengstenberg, and R. Biesinger. 1979. Optomotor control of wing beat and body posture in Drosophila. Biol. Cybern. 35: 101-112.

Greenspan, R.J. 1995. Flies, genes, learning, and memory. Neuron 15: 747-750.

Gronenberg, W. 1986. Physiological and anatomical properties of optical input fibres to the mushroom body in the bee brain. J. Insect Physiol. 32: 695-704.

Guo, A.K., L. Liu, S.-Z. Xia, C.-H. Feng, R. Wolf, and M. Heisenberg. 1996. Conditioned visual flight orientation in Drosophila: Dependence on age, practice, and diet. Learn. \& Mem. 3: 49-59.

Hammer, M. and R. Menzel. 1995. Learning and memory in honeybees. J. Neurosci. 15: 1617-1630.

Heide, G. and K.G. Götz. 1996. Optomotor control of course and altitude in Drosophila melanogaster is correlated with distinct activities of at least three pairs of flight steering muscles. J. Exp. Biol. 199: 1711-1726.

Heisenberg, M. 1989. Genetic approach to learning and memory (mnemogenetics) in Drosophila melanogaster. In Fundamentals of memory formation: Neural plasticity and brain function (ed. H. Rahmann), pp. 3-45. G. Fischer, Stuttgart, Germany.

1994. Central brain function in insects: Genetic studies on the mushroom bodies and central complex in Drosophila. In Neural basis of behavioural adaptations (ed. K. Schildberger and N. Elsner), Fortschritte der Zoologie 39, pp. 61-79. G. Fischer, Stuttgart, Germany.

Heisenberg, M. and R. Wolf. 1984. Vision in Drosophila (Genetics of microbehavior). In Studies of brain function (ed. V. Braitenberg, H.B. Barlow, T.H. Bullock, E. Florey, O.j. Grüsser, and A. Peters), 12, pp. 1-250. Springer, Berlin, Germany.

Heisenberg, M., A. Borst, S. Wagner, and D. Byers. 1985. Drosophila mushroom body mutants are deficient in olfactory learning. J. Neurogenet. 2: 1-30.

Helfand, S. and J. Carlson. 1989. Isolation and characterization of an olfactory mutant in Drosophila with a chemically specific defect. Proc. Natl. Acad. Sci.

86: 2908-2912.

Kim, Y.K., H.R. Koepfer, and L. Ehrmann. 1996.
Developmental isolation and subsequent adult behavior of Drosophila paulistorum: III. Alternative rearing. Behav. Genet. 26: 27-37.

Reichardt, W. and A. Guo. 1986. Elementary pattern discrimination (behavioural experiments with the fly Musca domestica). Biol. Cybern. 53: 285-306.

Rodrigues, V. 1988. Spatial coding of olfactory information in the antennal lobe of Drosophila melanogaster. Brain Res. 453: 299-307.

Rodrigues, V. and E. Buchner. 1984. $\left[{ }^{3} \mathrm{H}\right] 2$-Deoxyglucose mapping of odor-induced neuronal activity in the antennal lobes of Drosophila melanogaster. Brain Res. 324: 374-378.

Schuster, S. 1996. "Repräsentation visueller Objekte beim Suchlauf der Fliege Drosophila." Ph.D. thesis, University of Tübingen, Germany.

Schuster, S. and K.G. Götz. 1994. Adaptation of area covering random walk in Drosophila. In Proceedings of the 22nd Göttingen Neurobiology Conference 1994 (ed. N. Elsner and H. Breer), p. 304. Thieme, Stuttgart, Germany.

Siddiqi, O. 1991. Olfaction in Drosophila. In Chemical senses vol. 3; genetics of perception and communications (ed. C.J. Wysocki and M.R. Kare), pp. 79-96. Marcel Dekker, New York, NY.

Stocker, R.F. 1994. The organization of the chemosensory system in Drosophila melanogaster: A review. Cell Tissue Res. 275: 3-26.

Tully, T. 1996. Discovery of genes involved with learning and memory: An experimental synthesis of Hirschian and Benzerian perspectives. Proc. Natl. Acad. Sci.

93: $13460-13467$

Tully, T., V. Cambiazo, and L. Kruse. 1994a. Memory through metamorphosis in normal and mutant Drosophila. J. Neurosci. 14: 68-74.

Tully, T., T. Preat, S.C. Boynton, and M. Del Vecchio. 1994b. Genetic dissection of consolidated memory in Drosophila. Cell 79: 35-47.

Wolf, R. and M. Heisenberg. 1991. Basic organization of operant behaviour as revealed in Drosophila flight orientation. J. Comp. Physiol. A 169: 699-705.

Yin, J.C.P., M. Del Vecchio, H. Zhou, and T. Tully. 1995. CREB as a memory modulator: Induced expression of dCREB2 activator isoform enhances long-term memory in Drosophila. Cell 81: 107-115.

Received February 3, 1997; accepted in revised form February 18, 1997.

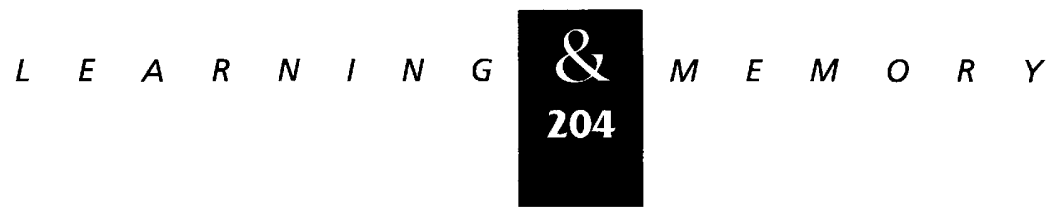




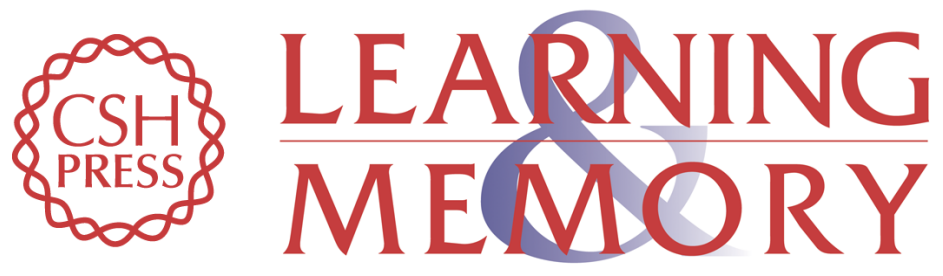

\section{Association of visual objects and olfactory cues in Drosophila.}

A Guo and K G Götz

Learn. Mem. 1997, 4:

Access the most recent version at doi:10.1101//m.4.2.192

References This article cites 39 articles, 10 of which can be accessed free at: http://learnmem.cshlp.org/content/4/2/192.full.html\#ref-list-1

License

Email Alerting Receive free email alerts when new articles cite this article - sign up in the box at the Service top right corner of the article or click here. 